\title{
Review of: "Addictive disorder"
}

\author{
Antony Moss 1 \\ 1 London South Bank University
}

Potential competing interests: The author(s) declared that no potential competing interests exist.

I think this is a very useful definition. My only query would be around the term 'chronic' - is this intended to refer to the length of an 'episode' of the disorder, or in the more usual sense of 'chronic relapsing disorder'? Either meaning is, I think, problematic certainly the latter (chronic relapsing) isn't true for many. Chronic in the sense of long-lasting is probably less contentious, but I still feel perhaps superfluous for the purposes of definition. 